\title{
A!
}

This is an electronic reprint of the original article.

This reprint may differ from the original in pagination and typographic detail.

Addad, Rami; Cadette Dutra, Diego; Bagaa, Miloud; Taleb, Tarik; Flinck, Hannu

\section{Towards A Fast Service Migration in 5G}

Published in:

2018 IEEE Conference on Standards for Communications and Networking, IEEE CSCN 2018

DOI:

$10.1109 / C S C N .2018 .8581836$

Published: 01/10/2018

Document Version

Peer reviewed version

Please cite the original version:

Addad, R., Cadette Dutra, D., Bagaa, M., Taleb, T., \& Flinck, H. (2018). Towards A Fast Service Migration in 5 G. In 2018 IEEE Conference on Standards for Communications and Networking, IEEE CSCN 2018 (IEEE

Conference on Standards for Communications and Networking). IEEE.

https://doi.org/10.1109/CSCN.2018.8581836

This material is protected by copyright and other intellectual property rights, and duplication or sale of all or part of any of the repository collections is not permitted, except that material may be duplicated by you for your research use or educational purposes in electronic or print form. You must obtain permission for any other use. Electronic or print copies may not be offered, whether for sale or otherwise to anyone who is not an authorised user. 
This is the accepted version of the original article published by IEEE.

(C) 2018 IEEE. Personal use of this material is permitted. Permission from IEEE must be obtained for all other uses, in any current or future media, including reprinting/republishing this material for advertising or promotional purposes, creating new collective works, for resale or redistribution to servers or lists, or reuse of any copyrighted component of this work in other works. 


\title{
Towards A Fast Service Migration in 5G
}

\author{
Rami Akrem Addad ${ }^{1}$, Diego Leonel Cadette Dutra ${ }^{2}$, Miloud Bagaa $^{1}$, Tarik Taleb ${ }^{1}$ \\ and Hannu Flinck ${ }^{3}$ \\ ${ }^{1}$ Aalto University, Espoo, Finland \\ ${ }^{2}$ Federal University of Rio de Janeiro, Rio de Janeiro, Brazil \\ ${ }^{3}$ Nokia Bell Labs, Espoo, Finland
}

\begin{abstract}
The development of the 5G technology has been driven by the need for faster and higher-capacity networks that would be able to sustain modern, high-demanding applications. Low-latency communication $(1 \mathrm{~ms}-10 \mathrm{~ms})$ is one of the important requirements of the 5G systems [1]. For achieving such requirements, the services should be shifted towards the vicinity of the users as much as possible. In this scope, Multi-access Edge Cloud (MEC) [2] will play a tremendous role in 5G technology by hosting various services close to the end-users. MEC is a network architecture concept that allows placing different services near the user equipment (UEs) for enabling very low latency and high bandwidth, which is required by real-time applications, such as mobile broadband in vehicles that are characterized by high UEs mobility. In order to ensure the service requirement in terms of low-latency communication, those services should follow (i.e., service migration) the user mobility by placing them always at the closest MEC. Placing services nearby highly mobile UEs is a very challenging problem. Lightweight migration of service states among MECs is one of the promising techniques that will mitigate such a problem. Motivated by the evolution of real time applications, we propose and evaluate three different mechanisms to improve the end user experience by using container-based live migration paradigm. In these approaches, we leverage the followme edge concept for enabling lightweight live migration. While the two first solutions take into consideration the mobile users' paths, e.g., cars, paths, the third one is oblivious to the users' paths. The obtained results demonstrate the efficiency of these solutions compared to prior works. These results show a drastic decrease in the service downtime during the migration and a near seamless user experience.
\end{abstract}

\section{INTRODUCTION}

The mobile network traffic keeps increasing at a very fast pace, and that is due to the emerging mobile applications, such as high-resolution video streaming, cloud gaming and augmented reality applications [3]. Within few years, $4 G$ systems definitely will not catch up with the pace of the traffic increase, as well as the expected requirements of the new emerging applications, such as autonomous cars, unmanned aerial vehicles (UAVs) and augmented reality. For this reason, many efforts, by both academia and industrial researchers, have been carried out in order to make the $5 G$ system a reality in the nearest future. As mentioned in [4], currently, there is a wide consensus among different actors about the services that should be enabled by $5 G$ systems. In fact, the $5 G$ system will not be an incremental version of the $4 G$ system with more capacity (i.e., new spectrum bands and higher spectral efficiencies) but will also target new emerging applications that will serve various industrial verticals. As in [4], 5G mainly focuses on enabling three verticals, each of which has different requirements.

1) Massive Machine-Type Communications (mMTC): Within this vertical, we expect up to tens of billions of network-enabled devices would be connected through $5 G$ systems. In this case, the $5 G$ system should ensure a scalable connectivity for those devices regardless of their locations (indoor or outdoor);

2) Ultra-reliable Machine-Type Communications (uMTC): This vertical requires ultra-reliable and low-latency communications (URLLC). The main applications, targeted by this vertical, are vehicle to anything (V2X) communication, industrial control applications and Smart Grid;

3) Extreme Mobile BroadBand (xMBB): This vertical is also refereed to as enhanced mobile broadband (eMBB). It mainly requires both extremely high data rates and lowlatency communication.

There is also a consensus among academia and industrials that the $5 G$ system will leverage emerging technology, such as network function virtualization (NFV) and software defined networking (SDN), for achieving its objectives [5]. While SDN gives more flexibility for connecting different components and enables the network softwarization, NFV allows running Virtual Network Functions (VNF) as software components on top of a virtualization system (i.e., Virtual Machines - VMs - or Containers) hosted in various clouds; allowing high flexibility and elasticity to deploy network services and functions [6]. These VNFs will run on top of cloud computing that are sparsely distributed over the globe. Cloud computing [7] offers cost-effective services, scalability features, and multi-tenancy support. The use of NFV on top of cloud computing in the $5 G$ system will reduce dramatically both capital expenditures (CAPEX) and operating expenses (OPEX) [8] [9]. However, it can be against the requirements of $5 G$ systems in terms of high data rates and low latency. In fact, instantiating different VNFs at faraway clouds would have a negative impact on both the data rates and end-to-end delay. In order to overcome this limitation, the concept of Multi-access Edge Computing (MEC) has been introduced [10]. It allows instantiating various VNFs (i.e, containers) in the vicinity of users. Clearly, the closer VNFs to the end users are, the higher data rates and 
lower latency we get. Indeed, the MEC technology allows the deployment of a subset of cloud resources at the edge of the cellular network (eNodeB), thus network congestion is reduced and better QoS/QoE [11] can be ensured for different customers. It also allows easy and efficient deployment of services by offloading compute-intensive applications from the core to the edge of the cloud.

The recent studies have confirmed the benefits of MEC on the cloud computing for achieving the objectives of the $5 G$ system [12]. In this context, MEC has been proven to be beneficial in reducing latency and offering an overall better experience. As mentioned earlier, a user in $5 G$ can be characterized by high mobility that may take him far away from the original MEC node where his service started running. In order to overcome such problem, a new concept, dubbed Follow Me Cloud (FMC) [13]-[17], has been introduced. In fact, the FMC concept allows the mobility of services between different edges for placing them closest to end users, which ensures low latency $(1 \mathrm{~ms}-10 \mathrm{~ms})$ and high capacity (more than $100 \mathrm{mbps})$. However, the main challenging problem of FMC is the service interruption during the migration of services from an edge cloud to another, which dramatically affects the requirements of industrial verticals.

In contrast to state-less migration [18]-[20], stateful migration, while ensuring service continuity after the mobility of the service to the new mobile edge, is a highly challenging problem. Towards addressing the problem of service interruption when migrating services between edge clouds, in this paper, we propose and evaluate three solutions that leverage on the container technology to reduce the migration time, and then reduce the services' interruption. The three solutions leverage LXC container tool and Checkpoint/Restore in Userspace (CRIU) for enabling stateful migration, and then ensuring the service continuity after migration. These proposed solutions enable the FMC concept for ensuring high availability and ultra-low latency for real-time applications, such as autonomous cars driving and UAVs' management [21]. While the first two solutions assume that the trajectories of the mobile users, e.g., cars or UAVs, are well known a priori. The third solution is more general, as it is oblivious to the mobile users' trajectory. The main contributions of this paper are as follows:

- Design and implementation of three container migration approaches using CRIU and LXC;

- To the best knowledge of the authors, the best downtime achieved by existing solutions is around $2 s$. In this paper, we have succeeded to reduce the downtime to be around $1 s$;

- A preliminary analysis of the impacts of CRIU's page server on the migration performance.

The remaining of the paper is organized as follows. Section II presents the background of this research and some related work. In Section III, we describe the types of migration evaluated in this paper and how they are deployed in our test environment. In Section IV, we present and discuss the results of our experimental evaluation. Finally, the paper concludes in Section V.

\section{BACKGROUND \& RELATED WORK}

This section introduces several underlying concepts that ease the paper readability. A summary of past relevant research work is also included.

\section{A. Background}

1) Containers: In the proposed solution, we leverage the container technology instead of the legacy Virtual Machine (VM) technology. Linux containers are a lightweight method for operating system virtualization that leverages kernel sharing with the host. Each container has its own environment comprising CPU, memory, I/O blocks, network resources, and resource management mechanisms. Most of the components needed by Linux containers are provided by the Linux kernel, for example, namespaces and SELinux that are used to ensure proper isolation between processes, and separation between the containers, and between the host and the containers.

2) Linux Container ( $L X C)$ : LXC is a lightweight virtualization technology integrated into Linux kernel to enable the running of multiple containers on the top of the same host. As opposed to OpenVZ [22], it runs on an unmodified kernel and allows Linux users to create and manage containers through its application program interface (API). LXC is a lightweight software virtualization tool that is built by mixing the Linux namespaces and CGroups to ensure a soft separation without virtualizing the hardware as the legacy virtual machine does. Compared to Docker, LXC [23] is a system level container. In other words, it provides a better flexibility when it comes to using system utilities, ensuring a similar powerful Linux system with a less overhead compared to a legacy virtual machine.

3) Checkpoint/Restore In Userspace (CRIU): To carry an efficient container-based service migration, the proposed system leverages the CRIU (Checkpoint/Restore In Userspace) tool [24], which allows to check-point/restore processes in Linux systems. It has the ability to save the state of a running application, so that its execution can later be resumed from the time of the checkpoint. Our migration approach for LXC containers with CRIU can be divided into two steps, namely checkpoint and restore, in addition to the copy process if the dumped files do not reside on a shared file system between the source and target host.

4) Live Migration: Compared to the cold migration and the hot migration that do not allow the copy of the memory pages to the destination host while it is in running state, the live migration is the process that guarantee the transfer of both the disk and the current memory pages when the migration is triggered.

5) Iterative Migration: As explained above, the migration has essentially two phases: $(i)$ copying the disk, $(i i)$ and then copying the page memory. Furthermore, while the first phase (disk copy) does not stop the state of the container, the second 
phase stops it. Thus, if the size of the memory pages is large, a critical downtime will be observed. To cope with this issue, the concept of iteration was created: in general, the copy of memory pages will be divided into several steps, each one of them (except the last one) does not stop the container and takes only the changes relative to the previous iteration. We name each intermediate step by predump phase, while the last one is named "dump phase". The dump phase will provoke the stop of the instance of virtualization (i.e., container in our case). However, a small downtime will occur due to the small number of memory pages copied to the destination host.

6) Downtime: Downtime is the period during which the services provided by the migrating instance (i.e., VM or container) are not available or no longer meet user requests. This notion plays a major role in high availability systems because this metric defines the power of the designed system offered to the users. Formally, the downtime is the exact time of the dump phase.

7) Total migration time: The total migration time is the period of time between the launch of the migration process and the moment when the instance is made available to the destination server. In other words, it is the sum of $(i)$ the downtime (i.e., dump), ( $i i)$ the time needed for the memory copy (i.e., predump) and disk copy. The total time $\Psi$ can be defined as follows:

$$
\Psi=\Gamma+\sum_{i=0}^{k} \gamma_{i}+\Upsilon
$$

where $\Gamma$ denotes the time needed to copy the disk, $k$ denotes the number of predump phases, $\gamma_{i}$ is the time needed during the predump $i$, and $\Upsilon$ is the time needed for the dump phase (i.e., Downtime) and the restore phase.

8) Checkpoint: The checkpointing procedure consists in collecting and saving the complete state of all processes running in the container. This action occurs in the last iteration (i.e., dump phase) in case of iterative migration or in the initial copy of memory pages in case of basic live migration. After that, all processes will be killed and will be waiting for a new upcoming action which is the restore.

9) Restore: It can be viewed as the opposite action of checkpointing. The restore procedure creates new processes from the dumped file which was obtained from the previous checkpoint action. After resuming processes execution and enabling the network, the container continues its normal execution.

\section{B. Related work}

The virtualization industry is moving towards supporting more and more elastic cloud-based solutions. In this context, container technology is gaining more ground than ever, thanks to its efficiency and ease of use. Unlike traditional VMs, containers do not run a full copy of an operating system, nor the underlying virtual hardware. In [25], containers and VMs were compared based on high availability. Results show that containers have better high availability (HA) features than hypervisor-based systems.

Yang [26] presented a generic checkpoint/restore mechanism and evaluated its performance using Docker. Each of the checkpoint and restore phases took $2183 \mathrm{~ms}$ and $1998 \mathrm{~ms}$, respectively, when considering a $256 M B$ container size. In contrast to Docker, LXC container gives more flexibility for running different applications, services and protocols. Indeed, Dockers are standalone applications running in an isolated environment and do not offer any system level functionality. Moreover, the checkpoint and restore phases take a considerable amount of time for a lightweight container. For this reason, in this paper, we have used LXC container technology instead of Docker technology.

Xavier et al. [27] evaluated the performance of a MapReduce cluster over LXC. Their experimental results showed that LXC offers a very good balance between performance and flexibility. Meanwhile, a previous comparison had shown that checkpoint/restart migration on OpenVZ had poor performance when compared to LXC [28], regarding the Downtime and Total Migration Time.

In [29], the authors evaluated container migration on a MEC platform based on OpenVZ. Their results showed that the total time for migration of a blank container is considerable even when using shared storage (NFS) and shared-async mode with a range of $(10 s-11 s)$. Machen et al. [30] presented a multilayer framework for migrating active applications in the MEC. While the obtained results show exceptional total migration times, the downtime was considerable with an average of $2 s$ in case of a blank container. The increase of the Downtime is due to the non-use of the iterative approach in the live migration process.

Thanks to the use of LXC combined with our new migration strategies based on iterative migration, the proposed solutions, herein, enhance the aforementioned works in terms of migration time, as well as the Downtime.

\section{ON USING LIGHTWEIGHT CONTAINERS IN MEC ENVIRONMENTS FOR ENABLING UMTC AND XMBB APPLICATIONS}

In this section, we first present the main architecture of our proposed framework for enabling lightweight container migration. Then, we will present the three proposed solutions that will enable lightweight container migration.

\section{A. Main architecture and problem formulation}

Fig. 1 depicts a typical three-layer cloud-based architecture for $5 \mathrm{G}$ networks. It supports scalable, distributed deployment models that aim to meet the $5 \mathrm{G}$ requirements, in terms of low latency and high data rate, for new mobile broadband and IoT services. The top layer (Layer 3 in Fig. 1) consists of the core network which can include data centers with powerful computing power from different vendors (e.g., Amazon, Microsoft, OpenStack). Orchestrated by the top layer, the edge clouds feature the Radio Access Network (RAN) with high spectral efficiency and bandwidth. This distributed computing model 
allows users - from the third layer - to be close to the compute capabilities according to their mobility. In our presented usecases, the users are on board high-speed vehicles or UAVs and their paths can be either pre-determined/predictable [31] (i.e., this allows us to trigger the migration process earlier on, before reaching the edge of the cell), or random which means that they can take a random path at any given moment and the actual migration has to be done in a limited time frame (when reaching the edge of the cell). The main focus is on the implementation of the live migration itself in several aspects to ensure a seamless migration across edge clouds, without taking into account other use-case-specific aspects, such as the signal strength received by each vehicle, user equipment (UE) or UAV.

\section{B. State-full service migration based on predefined path}

In this section, we present two solutions that should be used for predefined paths. A common use-case for this would be a vehicle or a UAV that has a known path from departure to destination. Knowing the paths allows us to anticipate the different source and target MECs for any migration along the way of the mobile node. This also means that the different MECs can be fully independent, e.g., no shared storage is needed, and gives enough time to copy the file system, which gives more freedom to the migration service. In the following section, we will introduce a live iterative migration based on two different approaches by using the CRIU tool [24].

1) Temporary File System based Lightweight Container Migration: This solution is also named tmpfs migration. The tmpfs migration solution starts first copying the container's file system along with the user files from the current MEC host to the destination MEC node using the rsync utility without service disruption. Second, the memory of the container is iteratively copied from the source MEC host to the destination MEC host. In this step, the CRIU utility will be used for iteratively dumping the container's memory - while it is running - into a tmpfs-mounted directory at the source MEC host. In this case, at the source MEC host, we have one reading from the memory and one writing to the tmpfs-mounted directory. In fact, we have one reading and one writing at the source MEC host. Each dump is then copied to the destination host via the network into the tmpfs-mounted directory at the destination MEC host which will result in a writing operation at the destination MEC host. Finally, read actions will be used in order to restore the container at the destination MEC host. We will also have one reading and one writing at the destination MEC host.

2) Disk-less based Lightweight Container Migration: In the first solution, we noticed that the memory images have two readings and two writings, which could have a negative impact on the total migration time. The process can be worse if the application uses a large amount of memory and/or multiple iterations (predumps). The disk-less migration solution overcomes this limitation. It aims to eliminate the step of copying the images to the local tmpfs directory in order to reduce further the total migration time. The proposed solution starts by copying the file system and preparing a tmpfs mount on both (source and destination) MEC hosts. Moreover, at the destination MEC host, we start a page server indicating the images directory and the port which will be used by the source MEC host to copy the files. Then on the source MEC host, using CRIU, we have adopted a new strategy by combining our iterative approach of live migration with the page server implementation. We start by dumping the memory pages directly into the target cloud using the two extra parameters: the page server's address and port while keeping the iterative concept working. Finally, we copy the rest of the images to the destination MEC host and we restore our container right after.

In order to test the two types of migration procedures as introduced above, we built a testbed, as shown in Fig. 2(b), to guarantee the most common architecture in a real case. The testbed consists of two VM hosts; each one representing a different Edge Cloud (i.e., an independent Infrastructure as a Service - IaaS - provider). Our container host is running on top of the first VM.

\section{State-full service migration based on undefined path}

In most real-world applications, the service provider (cloud service provider) does not know the movement pattern of the users. For this reason, we suggest a more general solution that considers the paths of users are unknown a priori. In this case, the copy of the file system and memory from the source MEC host to the destination MEC host could be a challenging process. For this reason, in this solution, also named lightweight containers migration with a shared file system, we need to use an alternative, fast and efficient migration process.

To eliminate the need to copy files over the network during the migration phase, we stored the container's file system along with the system images in a shared storage pool. This means that all that had to be done was to iteratively unload the container's memory using CRIU on the source node and then restore the container to the target node immediately after. This approach uses more network resources, while reducing the total migration time for LXC.

For evaluating the third solution, we have used the testbed, depicted in Fig. $3(b)$, that consists of three VMs. The first $\mathrm{VM}$ is the source MEC host, whereas the second one is the destination MEC host. Meanwhile, the third VM is the Network File Storage (NFS) server that is used to store the containers' file-system. We choose NFS because it represents a standard file system sharing technique, and while it is not scalable as a distributed or parallel file system, it allows the evaluation of the impact that a shared file system has on our migration procedure with containers. Furthermore, in terms of performance overhead, it represents for our setup a lowerbound as a distributed file system would impose additional overheads for a small-scale deployment. In the testbed, we 


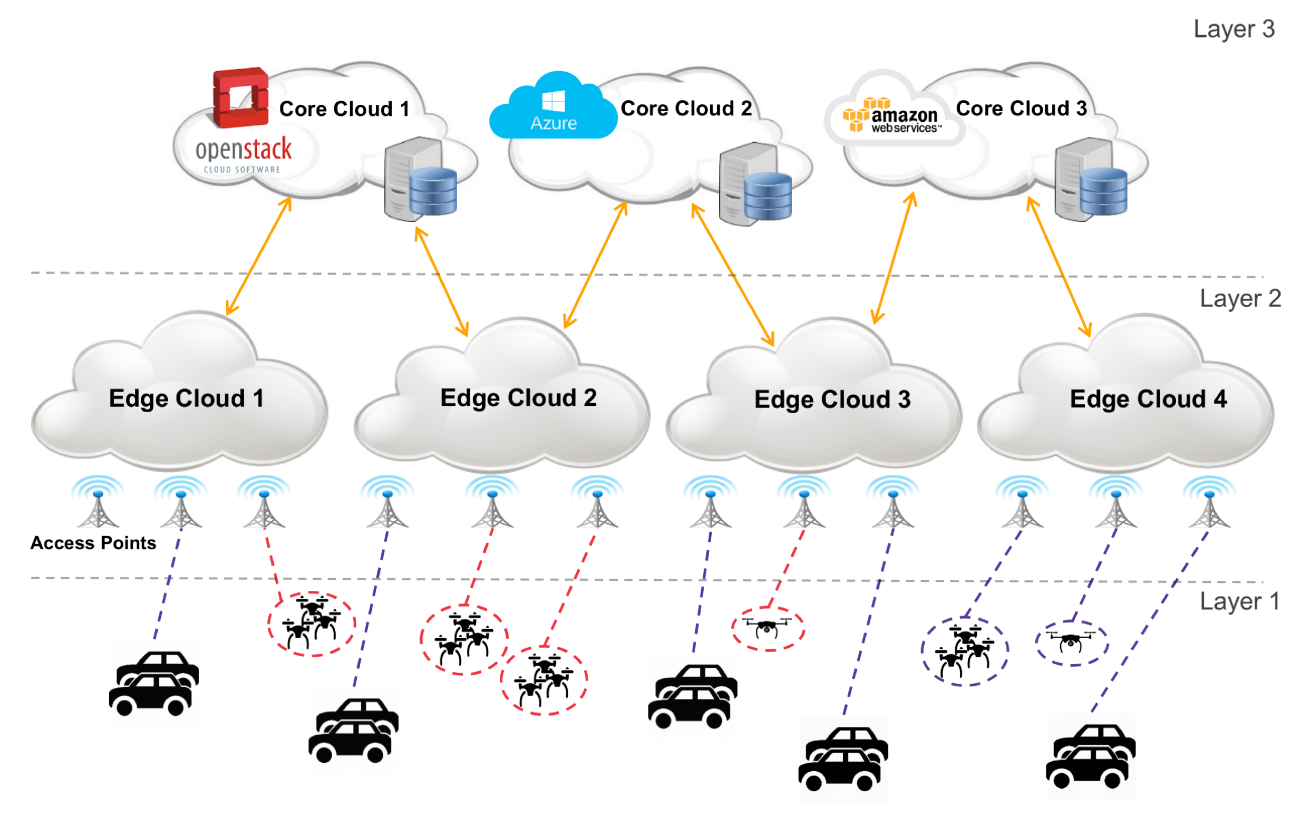

Fig. 1. A general architecture.

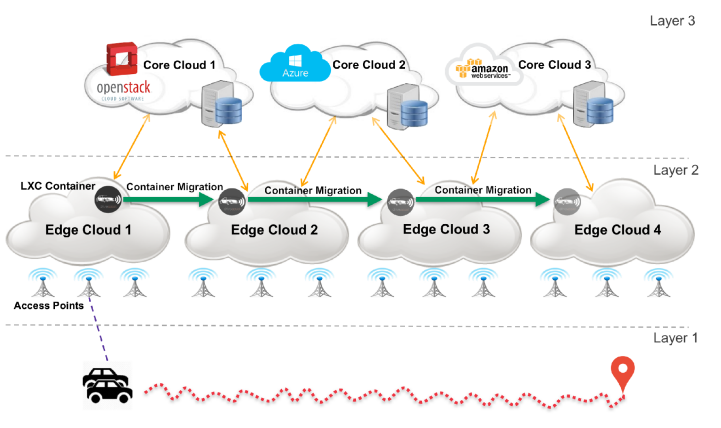

(a) Proposed architecture for known paths.

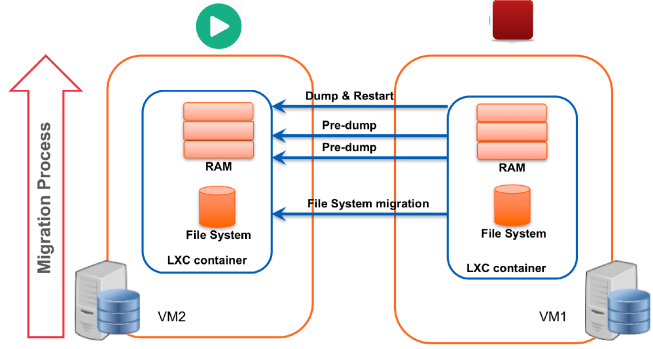

(b) Prototype testbed 1.

Fig. 2. Proposed solution for already known paths.

have ensured that the three VMs can communicate among themselves in order to enable container migration. While the communication between the MEC nodes and the NFS server is used for disk migration, the direct communication between MEC nodes is used for the migration of memory content.

\section{EXPERIMENTAL EVALUATION}

To evaluate the envisioned container migration scenarios, virtualized computer nodes are used. Each node uses Ubuntu 16.04 LTS with the 4.4.0-64-generic kernel, and has 4 core CPU and 4GB of main memory. The interconnection among the nodes is set at $1 \mathrm{~Gb} / \mathrm{s}$. The container environment was setup using LXC 2.8 and CRIU 2.6 (i.e., both are stable versions), while the NFS server used a dedicated virtual machine. For every container migration, we evaluate the total migration time and the container downtime as it directly corresponds to the application responsiveness/availability during the migration process.

TABLE I

TEST VIDEO SPECIFICATIONS

\begin{tabular}{l|c}
\hline Type & Configuration \\
\hline Codec & H.264 \\
Duration & $442 \mathrm{~s}$ \\
Bit rate & $1,560 \mathrm{Kbps}$ \\
Quality & $720 p$ \\
File size & $93.5 \mathrm{MB}$ \\
\hline
\end{tabular}

We conducted two sets of experiments; each was repeated ten times. The first one was a blank Linux container migration, with a file system size equal to $350 M B$. We kept an eye on the container's network reachability throughout the migration process in order to observe the impact caused by 


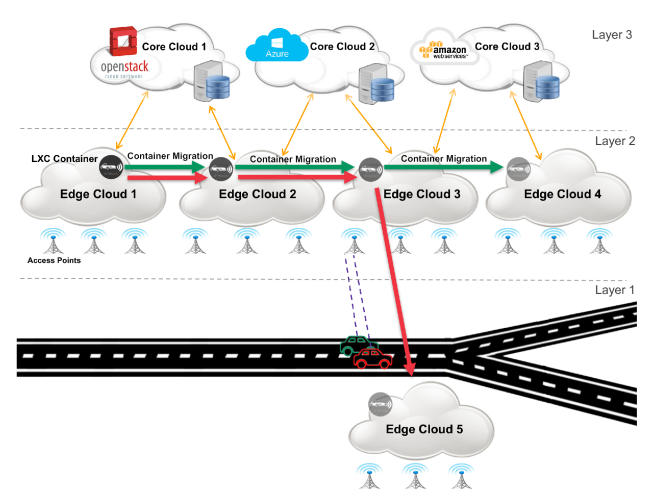

(a) Proposed architecture for unknown paths.

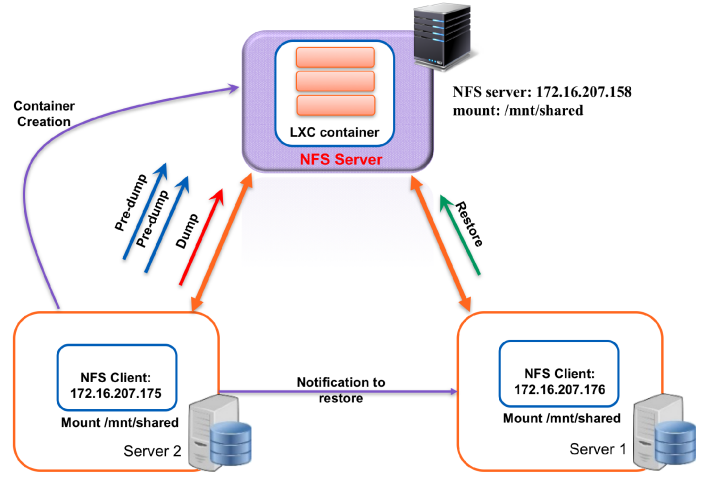

(b) Prototype testbed 2 .

Fig. 3. Proposed solution for unknown paths.

adding persistent data. The second one was the migration of a video streaming server NGINX running on top of a container, where file-system size was $590 M B$. The video used in our experiments has its configuration described in Table I.

\section{A. Migration induced downtime}

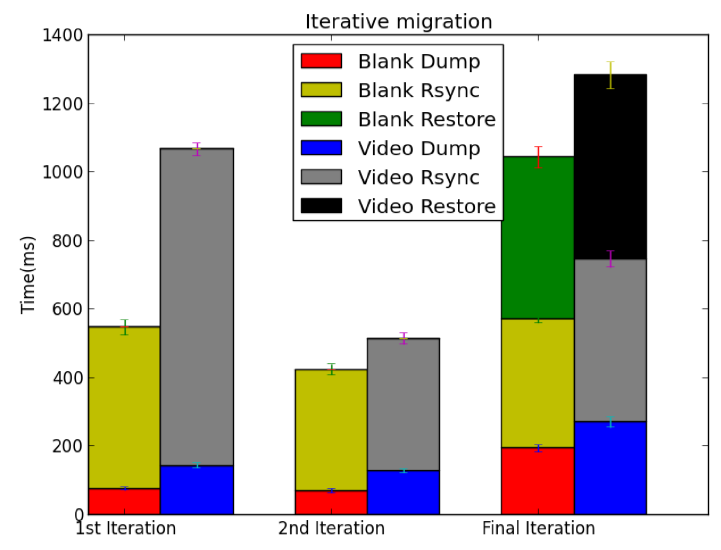

Fig. 4. Downtime comparison in case of an Iterative Migration process.

The first experiment uses the tmpfs migration strategy where the blank container's downtime, standard deviation and $95 \%$ C.I are $1042.974 \mathrm{~ms}, 52.790 \mathrm{~ms}$ and $39.806 \mathrm{~ms}$, respectively. Regarding the video-streaming container, the downtime, standard deviation and $95 \%$ C.I are $1282.012 \mathrm{~ms}, 76.141 \mathrm{~ms}$ and $57.415 \mathrm{~ms}$, respectively. As expected, the results for the video-streaming container are larger when compared to the blank container's results. The difference in these results is due to the additional copies of the network connections status and the NGINX internal control data to the target cloud. We also noticed that the addition of the NGINX HTTP server introduced more variability in our experiments, nevertheless, this represented an increase in the coefficient of variation of less than $15.686 \%$ going from 0.051 in the blank container to 0.059 in video streaming container.

Fig. 4 presents a breakdown of these times, alongside the breakdown of the initial phases of the migration procedure. The values presented in the figure are the mean and standard deviation of the times collected during the experiment for each sub-phase in the legend. As previously described, the migration procedures adopted for this paper use two precopy phases $-1^{\text {st }}$ and $2^{\text {nd }}$ iteration in the figure - before actually migrating the container to the destination compute node, in the final iteration phase. In the figure, each one of these iterations can be viewed in the $\mathrm{X}$-axis, while the $\mathrm{Y}$ axis presents the time in milliseconds. We also see that for both pre-copy phases, the video container took longer than the blank container, as the migration procedure needs to save and copy additional memory updates during each iteration, which ends up increasing the downtime in comparison with the blank container by $22.919 \%$.

Our second experimental scenario evaluates the Disk-less Migration described in Section III. As in our previous experiment, we will be using a blank container and a container running NGINX. The Disk-less Migration procedure imposed a $10.499 \%$ increase in the downtime for the blank container migration in comparison with the tmpfs Migration, which represents a mean downtime of $1152.476 \mathrm{~ms}$. The standard deviation for this experiment was $76.332 \mathrm{~ms}$ and $95 \%$ confidence interval was $57.558 \mathrm{~ms}$. As for the video container, the mean downtime was 1465.046 , which represents an increase of $14.277 \%$ over the blank container. The standard deviation for the video container Disk-less Migration was $107.117 \mathrm{~ms}$ and $95 \%$ confidence interval was $80.772 \mathrm{~ms}$. These results represented an increase in the coefficient of variation for the downtime of $10.606 \%$ when compared with the blank container going from 0.066 to 0.073 .

Fig. 5 presents a breakdown of the time for the 3 phases for the Disk-less Migration experiment scenario using the same $\mathrm{X} / \mathrm{Y}$-axes setup of the previous figure. As Fig. 5 shows, the increase in the downtime was caused by the increase of the 


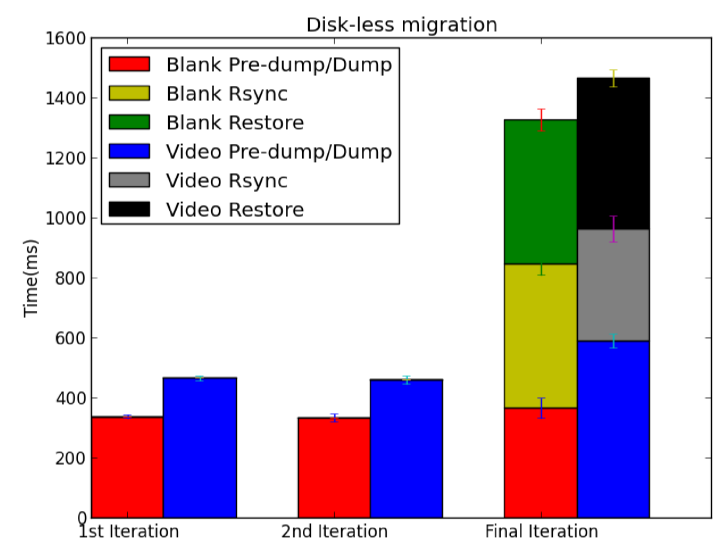

Fig. 5. Downtime comparison in case of a Disk-Less Migration.

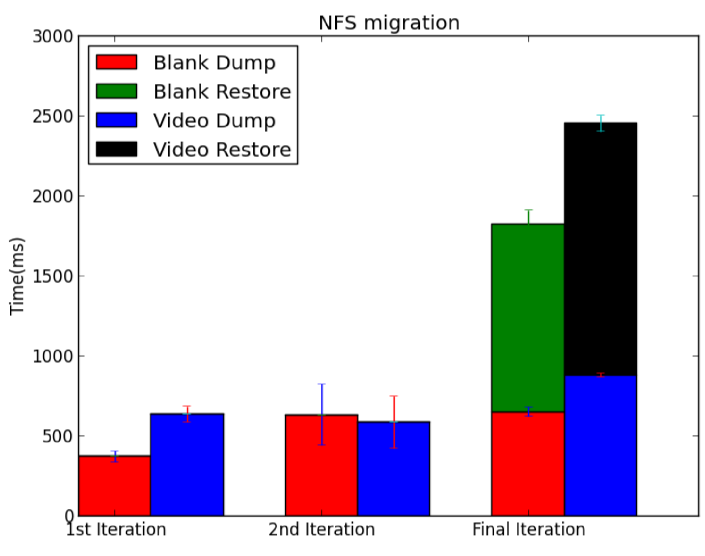

Fig. 6. Downtime comparison in case of a NFS migration.
Dump/PreDump sub-phase of the migration procedure. Our preliminary investigation suggests that this may be caused by the current implementation of the page server used in this experiment. As perceived in Fig. 4, Fig. 5 reveals that the video container took longer time to Dump/PreDump in comparison with the blank container. Furthermore, comparing the results presented in Fig. 4 and Fig. 5, it can be easily noticed for the blank container that in the pre-copy phases (i.e., $1^{\text {st }}$ iteration and $2^{\text {nd }}$ iteration) combination PreDump took more time than the same container in the Disk-less Migration, while the page server augmented PreDump takes longer time than the normal PreDump, which reinforce our suspicions about the page server implementation.

In the last experimental scenario, we evaluate the NFS migration described in Section III. As the previous scenarios, this evaluation is carried out with both a blank container and the video container. Our NFS migration imposed a $74.946 \%$ and $58.323 \%$ increase in the downtime for the blank container migration in comparison with the tmpfs Migration and the Disk-less Migration, respectively. This represents a mean downtime of $1824.64 \mathrm{~ms}$, while the standard deviation and the $95 \%$ confidence interval for this experiment were $122.657 \mathrm{~ms}$ and $57.558 \mathrm{~ms}$, respectively. The mean downtime for the video container was $2454.374 \mathrm{~ms}$, which represents an increase of $74.342 \%$ in comparison with the blank container. The standard deviation for the video container NFS migration was $72.592 \mathrm{~ms}$ and $95 \%$ confidence interval was $92.490 \mathrm{~ms}$.

In Fig. 6, we present the 3 phases of the NFS migration using the same $\mathrm{X} / \mathrm{Y}$-axis as before. Compared to the previous figures, the downtime increases due to the restore part of the final iteration. Our preliminary investigation suspects the network side because the target host restore procedure uses a remote file system which incurs an additional latency in the restore procedure. As perceived in Fig. 4 and Fig. 5, Fig. 6 shows that the video container took longer time to Dump/PreDump in comparison with the blank container. Furthermore, comparing the results presented in Fig. 4 and Fig. 5, the restore part takes much longer time in case of NFS and also the only manner to do the restore part is to use the target host, which reinforces our suspicions about the network side.

\section{B. Total migration time evaluation}

Our previous experimental results showed that our proposed approach reduces the downtime caused by the migration procedure. However, to enable its use for ultra-short latency services, we also need to address the total migration time. We addressed this evaluation using the same experimental scenarios of this section and plot the results in Fig. 7 for both the blank (red) and video (blue) containers. In Fig. 7, the Yaxis is in milliseconds and for each bar, we also plotted the $95 \%$ confidence interval of the mean.

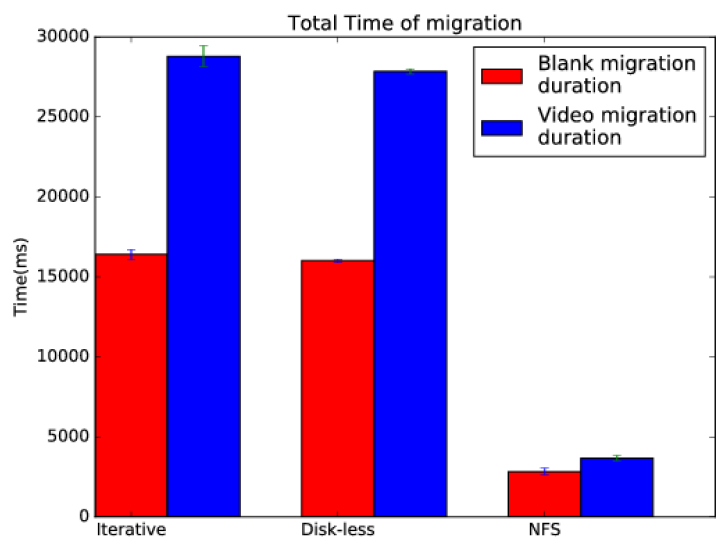

Fig. 7. Total migration time experienced in case of different approaches.

The mean total migration time for the blank container using the tmpfs migration method was $16,405.157 \mathrm{~ms}$ and the $95 \%$ confidence interval for this experiment was $312.089 \mathrm{~ms}$. 
For the video container, this migration method had a mean total migration time of $28,788.045 \mathrm{~ms}$ and $658.231 \mathrm{~ms}$ as its $95 \%$ confidence interval. The Disk-less Migration method imposed a mean total migration time of 16,015.2164 $\mathrm{ms}$ and 27,841.798 $\mathrm{ms}$ for both blank and video containers, respectively. Finally, for our shared storage scenario, the mean total migration time was $2,831.442 \mathrm{~ms}$ for the blank container with $95 \%$ confidence interval of $54.738 \mathrm{~ms}$, while in case of the migration of the video container, these values were $3,678.089 \mathrm{~ms}$ and $155.701 \mathrm{~ms}$, respectively.

In order to show the impact of adding services, we are focusing on empty containers to have a clear idea of the increase in migration time. From the results, we can observe that for tmpfs and Disk-less Migration, the long migration time was due to the file system copy, which was avoided in the NFS scenario. Furthermore, for the video streaming container, we clearly notice that the container size merely affects the total time of migration in the case of local storage because of the file system copy. Furthermore, for the NFS scenario, the longer migration time of the video container in comparison with the blank is due to the greater number of memory pages been copied.

\section{Impact of the number of pages on the migration downtime}

As our experimental evaluation results are influenced by the hardware technology available to us, e.g. network bandwidth, we summarized in Table II the number of memory pages copied during the last dump step in our migration procedures. In the first column, we have the evaluated scenario, followed by the mean number of pages copied during our evaluation, the standard deviation, and the $95 \%$ confidence interval. An initial assessment of this table shows us how disparate the results for the disk-less migration solution are in comparison with the others, which corroborates the results presented in Fig. 5, as it indicates that the larger downtime of this approach was caused by the interaction of the page server and the CRIU migration code which caused an increase in the number of copied pages by almost $x 8.84$ in our worst-case scenario.

TABLE II

SUMMARY OF PAGES COPIED DURING THE LAST DUMP.

\begin{tabular}{l|c|c|c}
\hline Migration types \& cases & Mean N. Pages & Std N. Pages & CI 95\% \\
\hline Blank-Tmpfs Migration & 509.5 & 6.3 & 5.14 \\
\hline Blank-Disk-less Migration & 1779.3 & 6.84 & 5.57 \\
\hline Blank-Shared file system Migration & 517.8 & 17.56 & 14.30 \\
\hline Video-Tmpfs Migration & 577.1 & 7.52 & 6.125 \\
\hline Video-Disk-less Migration & 5100.2 & 12.52 & 10.20 \\
\hline Video-Shared file system Migration & 551 & 16.8 & 13.68 \\
\hline
\end{tabular}

The behavior exhibited for the tmpfs and shared file system solutions are quite similar for both the blank container and our video streaming container, albeit their disparate downtime performances. As previously discussed, the higher downtime for the shared file system solution is caused by the multiple small writes over the network. Meanwhile, the tmpfs solution was able to drastically reduce the downtime as it keeps the number of copied pages in the last dump small and is able to fully utilize the network bandwidth through rsync. Furthermore, when we analyze the difference between the mean number of copied pages for the tmpfs and shared file system solutions for the blank container, and the same difference for the video streaming application, we conclude that the CRIU dump code exhibits a similar behavior for both solutions.

\section{Results Discussion}

As addressed in Table II, the tmpfs and shared file system solutions exhibit a similar behavior with regards to the number of copied pages during the last dump for both the blank container and the video streaming application. The analysis of the number of copied pages also enables us to do a qualitative assessment of the impact that the network bandwidth had over our experimental results, as Table II shows the average number of pages transferred in our best experimental scenario was 509.5 for the blank container and 577.1 for the video container. This means that on average, we transferred 2086912 bytes in the blank case, and 2281882 bytes in case of the video application, assuming the default $4 \mathrm{~KB}$ memory page used in our evaluation hardware. These transfers would take around $16.7 \mathrm{~ms}$ for the blank container, and $18.9 \mathrm{~ms}$ for the video container assuming a Fast Ethernet connection (100Mbps), while for a Gigabit Ethernet (1Gbps) the time spent copying the pages over the network was of the order of $1.67 \mathrm{~ms}$ for the blank container and $1.89 \mathrm{~ms}$ for the video container. This ten times millisecond improvement due to the network is still fifty times smaller than our improvements in downtime in comparison with Yang [26] and Machen et al. [30].

We have also observed that although the solutions based on predefined paths offer the best downtime results, the total migration time is the highest compared to the other ones. This is simply due to the fact that the file system, user files, and memory images are copied during the migration phase. This is not an issue since we can foresee the migration operations in advance. In contrast, the solution which is based on unknown paths offers great overall migration time, while sacrificing a few downtime milliseconds since the storage speed is limited by the available bandwidth of the network. However, this is the best solution for this scenario since the decision to trigger the migration along with the migration process itself has to be done in a matter of a couple of seconds.

\section{CONCLuSion}

In this paper, we proposed and evaluated three migration approaches for the container technology to enable the Follow Me Edge concept using MEC technology, ensuring high availability and supporting ultra-low latency for real-time applications, such as autonomous cars driving. The Follow Me Edge allows the system to guarantee a lower latency between the mobile user and the service provider, which is a fundamental requirement for $\mathrm{VN}$ and $5 \mathrm{G}$ networks. We have evaluated the proposed solution using real testbed experiments. The obtained results showed that while the NFS approach delivered the shortest migration time, it also imposed the 
highest downtime. Meanwhile, the larger migration time (in the approaches without a shared file system) was caused by the file system copy, which was done while the container was running. We have also shown that our approach that uses iterative migration achieved a mean downtime of $1,042.974 \mathrm{~ms}$, which represents an improvement of $61.039 \%$ in comparison to the work of Yang [26], and $50 \%$ better compared to Machen et al. [30].

Finally, it is important to note the high downtime of the Disk-less approach, which we expected to be the lowest downtime, in comparison with the iterative. Our preliminary analysis indicates a performance issue in the current implementation of CRIU's page server and its interaction with the page copying procedure as shown by the number of copied memory pages in Table II. In the future, we plan to extend our page server evaluation to improve its performance as this is a promising path to achieve sub-second downtime. We also intend to study the impact of the use of a distributed file system on the performances of the NFS approach.

\section{ACKNOWLEDGMENT}

This work was partially supported by the TAKE 5 project funded by the Finnish Funding Agency for Technology and Innovation (TEKES) and in part by the Finnish Ministry of Employment and the Economy. It is also partially supported by the European Unions Horizon 2020 Research and Innovation Program through the MATILDA Project under Grant No. 761898.

\section{REFERENCES}

[1] N. Alliance, "5g white paper," Tech. Rep., February 2015. [Online]. Available: https://www.ngmn.org/uploads/media/NGMN_5G_ White_Paper_V1_0.pdf

[2] H. Liu, F. Eldarrat, H. Alqahtani, A. Reznik, X. de Foy, and Y. Zhang, "Mobile edge cloud system: Architectures, challenges, and approaches," IEEE Systems Journal, vol. PP, no. 99, pp. 1-14, 2017.

[3] W. Cai, R. Shea, C. Y. Huang, K. T. Chen, J. Liu, V. C. M. Leung, and C. H. Hsu, "A survey on cloud gaming: Future of computer games," IEEE Access, vol. 4, pp. 7605-7620, 2016.

[4] "Preliminary views and initial considerations on 5G RAN architecture and functional design," Tech. Rep., March 2016. [Online]. Available: https://bscw.5g-ppp.eu/pub/bscw.cgi/d92532/5GPPP-METIS-II-5G-RAN-Architecture-White-Paper.pdf

[5] I. Afolabi, T. Taleb, K. Samdanis, A. Ksentini, and H. Flinck, "Network slicing; softwarization: A survey on principles, enabling technologies; solutions," IEEE Communications Surveys Tutorials, vol. PP, no. 99, pp. $1-1,2018$.

[6] F. Z. Yousaf and T. Taleb, "Fine-grained resource-aware virtual network function management for $5 \mathrm{~g}$ carrier cloud," IEEE Network, vol. 30, no. 2, pp. 110-115, March 2016.

[7] A. Ahmed and E. Ahmed, "A survey on mobile edge computing," in 2016 10th International Conference on Intelligent Systems and Control (ISCO), Jan 2016, pp. 1-8.

[8] T. Taleb, M. Corici, C. Parada, A. Jamakovic, S. Ruffino, G. Karagiannis, and T. Magedanz, "Ease: Epc as a service to ease mobile core network deployment over cloud," IEEE Network, vol. 29, no. 2, pp. 78-88, March 2015.

[9] T. Taleb, "Toward carrier cloud: Potential, challenges, and solutions," IEEE Wireless Communications, vol. 21, no. 3, pp. 80-91, June 2014.

[10] T. Taleb, K. Samdanis, B. Mada, H. Flinck, S. Dutta, and D. Sabella, "On multi-access edge computing: A survey of the emerging $5 \mathrm{~g}$ network edge cloud architecture and orchestration," IEEE Communications Surveys Tutorials, vol. 19, no. 3, pp. 1657-1681, thirdquarter 2017.
[11] H. Rifa, S. Mohammed, and A. Mellouk, "A brief synthesis of qos-qoe methodologies," in 2011 10th International Symposium on Programming and Systems, April 2011, pp. 32-38.

[12] Y. C. Hu, M. Patel, D. Sabellaa, N. Sprecher, and V. Young, "Mobile edge computing a key technology towards 5g," Tech. Rep., September 2015. [Online]. Available: http://www.etsi.org/images/files/ ETSIWhitePapers/etsi_wp11_mec_a_key_technology_towards_5g.pdf

[13] T. Taleb and A. Ksentini, "An analytical model for follow me cloud," in 2013 IEEE Global Communications Conference (GLOBECOM), Dec 2013, pp. 1291-1296.

[14] A. Ksentini, T. Taleb, and M. Chen, "A markov decision processbased service migration procedure for follow me cloud," in 2014 IEEE International Conference on Communications (ICC), June 2014, pp. $1350-1354$

[15] T. Taleb and A. Ksentini, "Follow me cloud: interworking federated clouds and distributed mobile networks," IEEE Network, vol. 27, no. 5 , pp. 12-19, September 2013.

[16] A. Aissioui, A. Ksentini, A. Gueroui, and T. Taleb, "On enabling $5 \mathrm{~g}$ automotive systems using follow me edge-cloud concept," IEEE Transactions on Vehicular Technology, vol. PP, no. 99, pp. 1-1, 2018.

[17] A. Ksentini, T. Taleb, and F. Messaoudi, "A lisp-based implementation of follow me cloud," IEEE Access, vol. 2, pp. 1340-1347, 2014.

[18] I. Farris, T. Taleb, M. Bagaa, and H. Flick, "Optimizing service replication for mobile delay-sensitive applications in $5 \mathrm{~g}$ edge network," in 2017 IEEE International Conference on Communications (ICC), May 2017, pp. 1-6.

[19] I. Farris, T. Taleb, A. Iera, and H. Flinck, "Lightweight service replication for ultra-short latency applications in mobile edge networks," in 2017 IEEE International Conference on Communications (ICC), May 2017, pp. 1-6.

[20] I. Farris, T. Taleb, H. Flinck, and A. Iera, "Providing ultrashort latency to usercentric $5 \mathrm{~g}$ applications at the mobile network edge," Transactions on Emerging Telecommunications Technologies, vol. 29, no. 4, p. e3169. [Online]. Available: https://onlinelibrary.wiley.com/doi/ abs/10.1002/ett.3169

[21] N. H. Motlagh, T. Taleb, and O. Arouk, "Low-altitude unmanned aerial vehicles-based internet of things services: Comprehensive survey and future perspectives," IEEE Internet of Things Journal, vol. 3, no. 6, pp. 899-922, Dec 2016.

[22] Virtuozzo, "Openvz," Tech. Rep., (Last visit on : 02-12-2017). [Online]. Available: https://openvz.org/Main_Page

[23] D. Bernstein, "Containers and cloud: From lxc to docker to kubernetes," IEEE Cloud Computing, vol. 1, no. 3, pp. 81-84, Sept 2014.

[24] team CRIU, "Criu (checkpoint and restore in user space) main page," 2016. [Online]. Available: https://criu.org/Main_Page

[25] Wubin, Li and Ali, Kanso, "Comparing Containers versus Virtual Machines for Achieving High Availability," in 2015 IEEE International Conference on Cloud Engineering, Tempe, AZ, 2015, pp. 353-358.

[26] Yang Chen, "Checkpoint and Restore of Micro-service in Docker Containers," in Proceedings of the 3rd International Conference on Mechatronics and Industrial Informatics.

[27] M. G. Xavier, M. V. Neves and C. A. F. D. Rose, "A Performance Comparison of Container-Based Virtualization Systems for MapReduce Clusters," in 2014 22nd Euromicro International Conference on Parallel, Distributed, and Network-Based Processing, Torino, 2014, pp. 299-306.

[28] P. S. V. Indukuri, "Performance comparison of Linux containers (LXC) and OpenVZ during live migration," Master's thesis, Blekinge Institute of Technology, Sweden, 2016.

[29] T. Taleb, S. Dutta, A. Ksentini, M. Iqbal, and H. Flinck, "Mobile edge computing potential in making cities smarter," IEEE Communications Magazine, vol. 55, no. 3, pp. 38-43, March 2017.

[30] A. Machen, S. Wang, K. K. Leung, B. J. Ko, and T. Salonidis, "Live service migration in mobile edge clouds," IEEE Wireless Communications, vol. PP, no. 99, pp. 2-9, 2017.

[31] A. Nadembega, A. Hafid, and T. Taleb, "A destination and mobility path prediction scheme for mobile networks," IEEE Transactions on Vehicular Technology, vol. 64, no. 6, pp. 2577-2590, June 2015. 